\title{
Revista Andina de Educación
}

\author{
http://revistas.uasb.edu.ec/index.php/ree
}

\section{Estudiantes de un plantel educativo secundario del sur del Ecuador y un Entorno Virtual de Aprendizaje (EVA): Impacto de su implementación}

\author{
Juan Urdiales Flores ${ }^{\mathrm{a}, *}$, Leonardo Armijos Bacuilima ${ }^{\mathrm{a}}$, Diego Urdiales ${ }^{\mathrm{a}, \mathrm{b}}$ \\ ${ }^{a}$ Unidad Educativa Herlinda Toral. Río Malacatus, 4 -312 y Altarurco. Cuenca, Ecuador. \\ ${ }^{\mathrm{b}}$ Universidad de Cuenca. Av. 12 de Abril y Av. Loja. Cuenca, Ecuador.
}

PUNTOS DESTACADOS

- La implementación de Entornos Virtuales de Aprendizaje (EVAs) en educación secundaria en el sur del Ecuador es de gran utilidad, sin embargo, existen brechas de conocimiento intrínsecas en su uso y estas deben suplirse para obtener mejores resultados de aprendizaje.

- Los beneficios de la implementación de los EVAs son muy conocidos, a pesar de esto, existen vacíos de conocimiento. Estos vacíos fueron evaluados a partir de la percepción de los estudiantes de educación secundaria en el Ecuador.

- Es imprescindible investigar el mejoramiento de dos componentes: Evaluación en Línea y Oportunidad de Mejoramiento del rendimiento Académico en un EVA.

INFORMACIÓN DEL ARTÍCULO

Historial del artículo:

Recibido el 01 de mayo de 2020

Aceptado el 21 de mayo de 2020

Publicado el 19 de junio de 2020

Palabras clave:

Entornos Virtuales de Aprendizaje (EVAs)

Percepción estudiantil

Educación secundaria

\section{RESUMEN}

El cambio tecnológico está alterando la forma en que los educadores entregan el contenido de las asignaturas. El crecimiento y la aceptación generalizada del Internet ha dado lugar a la creación de Entornos Virtuales de Aprendizaje (EVAs) en la enseñanza en cualquier nivel educativo. Para facilitar la integración de los EVAs, desde una perspectiva pedagógica, las nuevas tecnologías deben evaluarse en un esfuerzo por establecer si su introducción ha tenido un efecto beneficioso en los resultados del aprendizaje. Con ese fin, en la presente investigación se examinan las percepciones de los estudiantes de educación secundaria con relación a una serie de componentes y atributos de diseño de la plataforma virtual (por ejemplo, indicaciones de uso, evaluación en línea). Las respuestas de 64 estudiantes del Colegio Herlinda Toral de Cuenca, entre 17 a 18 años, revelan que la satisfacción y motivación está presente en el uso del EVA, sin embargo, los componentes de Evaluación en Línea y Oportunidad de Mejoramiento del Rendimiento Académico son los que obtienen porcentajes menores de aceptación. La identificación de estos factores claves proporcionan un punto de referencia útil para los educadores que contemplan la aplicación de un EVA en el Ecuador.

(c) 2020 Urdiales-Flores, Armijos-Bacuilima \& Urdiales CC BY-NC 4.0

\section{Introducción}

El crecimiento y la difusión de las Tecnologías de la Información y la Comunicación (TICs) ha desarrollado un interés masivo en cómo las computadoras, otros dispositivos y herramientas de internet pueden beneficiar y mejorar los procesos educativos y fomentar el uso de la tecnología en las aulas (Rahmany et al., 2014). Un recurso ofrecido por las TICs son los Entornos Virtuales de Aprendizaje (EVAs) conocidos también como Plataformas en Línea de Aprendizaje (PLA), Sistema del Manejo de Aprendizaje (SMA), o Sistema de Cursos de Aprendizaje (SCA). Las diferentes denominaciones pueden tener utilidades especificas en función del propósito de estudio. Nosotros utilizamos la definición de EVAs, adoptada en varios estudios (de Lange et al., 2003; Mueller \& Strohmeier, 2011; Vasquez et al., 2015).

\footnotetext{
*Autor principal: Juan Uridiales Flores. Unidad Educativa Herlinda Toral. Río Malacatus, 4 -312 y Altarurco. Cuenca, Ecuador. Correos electrónicos: juanurdys@gmail.com (J. Urdiales Flores), leonardo_armijos@hotmail. com (L. Armijos), dhurdiales@uc.cl (D. Urdiales).
}

Los EVAs son espacios en línea que maestros y estudiantes usan para presentar y compartir recursos, y planificar actividades (Edel-Navarro, 2009). Estas plataformas se pueden usar para enseñar un curso completo en línea o como apoyo para cursos presenciales. Los EVAs proporcionan un medio para gestionar la experiencia de aprendizaje, comunicar la experiencia de aprendizaje prevista y facilitar la participación de tutores y alumnos (Sneha y Nagaraja, 2014). Su objetivo principal es facilitar, motivar y proporcionar experiencias de aprendizaje que van más allá del aula. Autores como Barker y Peter (2013) afirman que los EVAs cuentan con un amplio espectro de investigaciones que muestran impactos positivos en diferentes contextos.

En América Latina, los EVAstieneun granaugea partir de la década de 1990 (Arroyo Vera et al., 2018); contemplando nuevos soportes para la aplicación de mensajes de texto, sonido e imágenes. En la región de América Latina, existirá un crecimiento en la utilización de plataformas virtuales de aprendizaje con gran énfasis en procesos semipresenciales de código abierto. En Ecuador, el aprendizaje remoto inicia 
en 1962 impartiéndose clases por radio la Fundación de las Escuelas Radiofónicas Populares (Arroyo Vera et al., 2018), el propósito principal era brindar educación a sectores de difícil acceso. Según Álvarez et al. (2015), en 1976, la Universidad Técnica comenzó la educación de modalidad abierta con poca presencia del alumno en aulas físicas. La implementación de EVAs en cualquier nivel de educación es de gran utilidad. Sin embargo, durante el proceso previo de implementación es imprescindible conocer las características necesarias que debe tener un EVA con la finalidad que estudiantes y profesores obtengan los mejores resultados. Varios estudios concuerdan que la motivación de los estudiantes en la utilización EVAs depende de la mediación pedagógica del maestro, la estructura del EVA y el compromiso del estudiante (Dyson et al., 2003; Virkus et al., 2009; Walker et al., 2005). El vacío de conocimiento, hasta la actualidad, demanda encontrar los componentes claves para la motivación de los estudiantes y así lograr disminuir la brecha de conocimiento en estudiantes (Álvarez et al., 2015; Mueller et al., 2011).

El presente estudio tiene como objetivo examinar la percepción de cinco variables del EVA, a saber: el suministro de indicaciones del uso, calidad de la evaluación en línea, oportunidad de mejorar su rendimiento, facilidad y utilidad, y aplicación de tecnología (por ejemplo, celular, tablet) en la evaluación general del suministro de recursos de aprendizaje en el marco de un programa de educación secundaria de la ciudad de Cuenca. La percepción de los estudiantes contribuirá al desarrollo, implementación y mejora/evaluación (continua) de los EVAs en el Ecuador.

Nuestra investigación tiene un diseño cuantitativo de tipo exploratorio, no pretendemos estimar un factor o patrón de comportamiento, sino más bien, establecer un punto de partida para conocer la percepción de los estudiantes de educación secundaria frente a un EVA y generar directrices que nos permitan dirigir nuevas investigaciones en este área de conocimiento.

\section{Materiales y método}

Los datos fueron recolectados a partir de encuestas realizadas en formularios de Google, y luego analizados e interpretados mediante en Microsoft Excel.

\subsection{Cuestionario de percepción estudiantil del EVA}

El cuestionario de percepción consta de 5 preguntas que abarcan los componentes principales de un EVA. La pregunta (i) ¿Cómo fue su experiencia con el EVA con respecto a las indicaciones de uso y anuncios?, está orientada a explorar la estructura de ayuda e indicaciones para que el estudiante sea capaz de utilizar el EVA de manera amigable y autónoma. La pregunta (ii) Califique la calidad de los simuladores con relación a la evaluación en línea, pretende obtener una descripción de la funcionalidad que tiene el EVA para proporcionar una calificación de forma automática al estudiante que le permita conocer su puntaje y retroalimentarse de forma inmediata. La pregunta (iii) ¿Cómo califica la oportunidad de mejorar su rendimiento académico mediante el EVA?, está direccionada a conocer la percepción de los estudiantes sobre la posibilidad de mejora de sus calificaciones y/o sus conocimientos. La pregunta (iv) ¿El EVA le pareció útil y fácil para su educación?, tiene como objetivo conocer la factibilidad y facilidad de uso de un EVA. Finalmente, la pregunta (v) ¿Cuál es su percepción al usar celular, tablet y/o computadora para un EVA?, es una exploración inicial de la percepción que tienen los estudiantes de las herramientas básicas e indispensables para implementar un EVA en la educación secundaria.

\subsection{Recolección de los datos}

Los cinco componentes del EVA presentes en el cuestionario fueron evaluados por los estudiantes respondiendo a las preguntas planteadas mediante una escala de percepción de 5 niveles (Excelente, Muy Bueno, Bueno, Regular, Malo), tipo Likert (cualitativa politómica ordinal) similar a las empleadas en los estudios de Lange et al. (2003) y Herrera Mosquera (2017). La encuesta se llevó a cabo tras la finalización del programa de estudios.

Este programa tuvo una duración de 9 meses, abarcando las temáticas de preparación de la prueba de Educación Superior del Ecuador, requisito necesario para ingresar a las Universidades. El EVA del programa de estudio fue desarrollado en Moodle. El programa de preparación fue impartido a 240 estudiantes de 6 aulas, todos los estudiantes cursaban tercero de bachillerato y sus edades oscilaron entre los 17 y 18 años.

Las preguntas siguieron los lineamientos de Hayes (2009). Además, la realización del cuestionario se planifico de forma independiente al seguimiento del cuso. Este hecho, junto con el anonimato, tenía la intención incentivar a la sinceridad y lograr una evaluación concreta y veraz sobre el alcance del EVA. Debido el anonimato de las preguntas, su evaluación e interpretación también se llevó de forma independiente. Sin embargo, para las conclusiones fue imperativo integrar cada uno de los componentes en una evaluación integral. Puesto que cada dimensión evaluada es parte de un todo, la evaluación debía ser holística.

\subsubsection{Confiabilidad del cuestionario tipo Likert (politómica ordinal)}

Para determinar la confiabilidad del cuestionario, calculamos el alfa de Cronbach $(\propto)$, que es una medida de fiabilidad de las encuestas realizadas en estudios en educación (Taber, 2018). La ecuación 1 describe el cálculo de $\propto$.

$$
\propto=\frac{K}{K-1}\left[1-\frac{\sum_{i=1}^{K} \sigma_{Y i}^{2}}{\sigma_{X}^{2}}\right]
$$

Donde:

$\propto=$ Alpha de Cronbanch.

$\mathrm{K}=$ Cantidad de preguntas, en nuestro caso cinco.

$\sigma_{Y_{\mathrm{i}}}{ }^{2}=$ Varianza de las puntuaciones de la pregunta $\mathrm{i}$.

$\sigma_{x}{ }^{2}=$ Varianza de las puntuaciones observadas de los individuos en nuestra investigación de las 64 respuestas de las encuestas.

El cálculo del alpha de Cronbanch $(\propto)$ fue de 0.86 , esto nos permite tener un alto grado de confiabilidad del cuestionario utilizado para conocer la percepción de los cinco componentes del EVA. 


\subsection{Características del muestreo}

Para el muestreo se seleccionó solo a aquellos alumnos que tenían una interacción completa con el EVA en términos de tiempo, es decir, solo a aquellos que iniciaron el curso y lo terminaron. Se excluyó la participación de alumnos que se retiraron del curso y la de aquellos que se unieron desde la mitad en adelante del programa. Se tomó esta decisión para que las respuestas dadas estuvieran en un contexto similar, en la forma que el EVA pasó a ser parte de su pedagogía. Las personas que se unieron de la mitad en adelante podrían tener una perspectiva distinta o generar discrepancia, dada solamente por el corto tiempo en el que estuvieron expuestos al EVA. En cambio, con los alumnos retirados existiría una dimensión muy separada, englobando las razones que llevaron a su separación del programa y si alguna de ellas está o no enlazada al uso del EVA. Siendo un análisis completo en sí, y con potencial para ser sujeto a una investigación propia, se decidió dejar de lado este tipo de alumnado.

El muestreo se realizó de forma no aleatoria por conveniencia y todas las encuestas fueron tomadas el mismo día. Medida necesaria para disminuir el sesgo del muestreo proveniente de influencias grupales y de carácter personal (amistad, compartir las preguntas, etc.). Además, las respuestas de las encuestas fueron de carácter voluntario (los estudiantes fueron notificados sin previo aviso y cada estudiante decidió si deseaba responder) y de forma personal en el centro de cómputo de la Unidad Educativa Herlinda Toral. Las muestras totales recogidas, de forma voluntaria, fueron 64 encuestas válidas para nuestra investigación. Hacemos hincapié en que, a pesar de que la muestra es relativamente pequeña con respecto al total de estudiantes que tomaron el curso, consideramos que los datos obtenidos son un gran aporte y tienen un notable valor, ya que actualmente resulta un reto obtener datos de esta índole de un plantel de educación secundaria fiscal.

\section{Resultados y discusión}

La Figura 1 describe los porcentajes de la experiencia percibida con relación a las indicaciones del uso del EVA. El $62.5 \%$ de los estudiantes responde que el EVA presenta una excelente experiencia mientras que el $18.8 \%$ y el 9.4 responde que la experiencia fue muy buena y buena, respectivamente. El $9.3 \%$ restante responde que la experiencia referida a las indicaciones de uso es regular o mala.

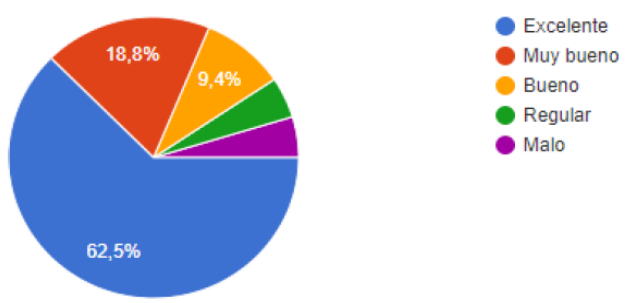

Fig. 1. ¿Cómo fue su experiencia con el EVA con respecto a las indicaciones de uso y anuncios?

Elaboración: Propia.

La Figura 2 representa la percepción sobre la calidad de los simuladores relativa a la evaluación en línea. Se observa que el $48,4 \%$ de los encuestados la considera excelente, el $28.1 \%$ muy buena, el $12.5 \%$ buena y el $10.9 \%$ regular. Ningún encuestado tiene una percepción mala para este componente.

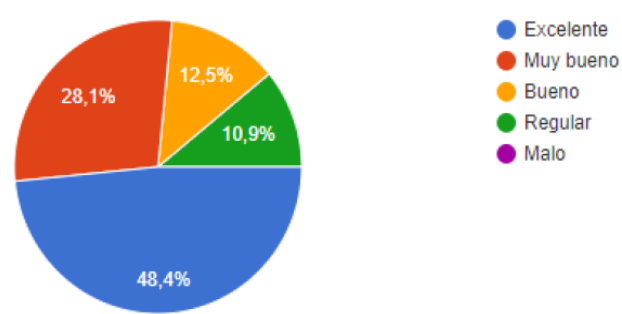

Fig. 2. Califique la calidad de los simuladores con relación a la evaluación en línea

Elaboración: Propia.

En la Figura 3 se presenta la percepción de los estudiantes concerniente a la oportunidad de mejorar su rendimiento académico. El $45.3 \%$ de los encuestados responde que es excelente, el $34.4 \%$ muy bueno, el $10.9 \%$ bueno; y el $9.4 \%$ regular. Ningún encuestado tiene una percepción mala para este componente.

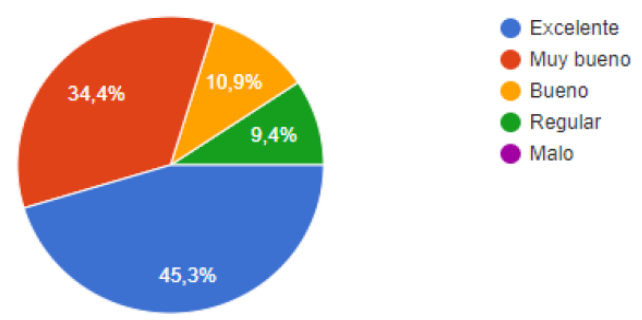

Fig. 3. ¿Cómo califica la oportunidad de mejorar su rendimiento académico mediante el EVA?

Elaboración: Propia.

La percepción de la utilidad y facilidad de uso del EVA se describe en la Figura 4. Se obtuvo que el $56.3 \%$ considera que el EVA es un entorno excelente. El 23.4\%, $12.5 \%$ y $7.8 \%$ de los encuestados respondieron que lo consideran muy bueno, bueno o regular, respectivamente. Ningún encuestado tiene una percepción mala en este componente.

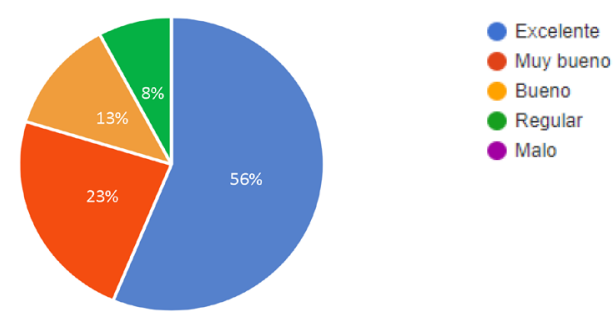

Fig. 4. ¿El EVA le pareció útil y fácil para su educación? 1: Malo; 2: Regular; 3: Bueno; 4: Muy Bueno; 5: Excelente.

Elaboración: Propia.

La percepción del uso de dispositivos electrónicos digitales se presenta en la Figura 5. El 64.1\% de los encuestados considera el uso de manera excelente. El 25\% de encuestados lo percibe como muy bueno. Finalmente, el $10.9 \%$ considera el uso de celular, tablet y/o computadora 
como regular. Ningún encuestado tiene una percepción mala para este componente.
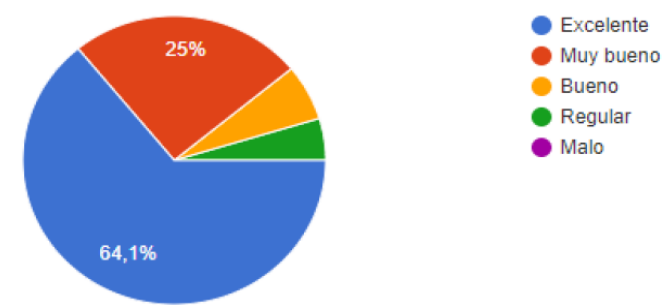

Fig. 5. ¿Cuál es su percepción al usar celular, tablet y/o computadora para un EVA?

Elaboración: Propia.

La Figura 6 representa la frecuencia de respuestas estudiantiles a las cinco preguntas. La tendencia de percepción para todas las preguntas está sesgada hacia la derecha, es decir, hacia muy bueno (rango de 12 a 22 votaciones estudiantiles) y excelente (rango de 29 a 41 votaciones estudiantiles). El rango de percepción en la categoría regular fluctúa de 3 hasta 7 votaciones estudiantiles. Es visible que la única respuesta estudiantil que obtiene la percepción malo corresponde a la pregunta 1 (¿Cómo fue su experiencia con el EVA con respecto a las indicaciones de uso y anuncios?) con la votación de 3 estudiantes. Además, en la categoría excelente, la pregunta 5 (¿Cuál es su percepción al usar celular, tablet y/o computadora para un EVA?) fue la que obtuvo mayores respuestas, con 41 votaciones, seguida de la pregunta 1 , con 40 votaciones, la pregunta 4 , con 36 votaciones, la pregunta 2, con 31 votaciones $y$, finalmente, la pregunta 3 , con 29 votaciones.

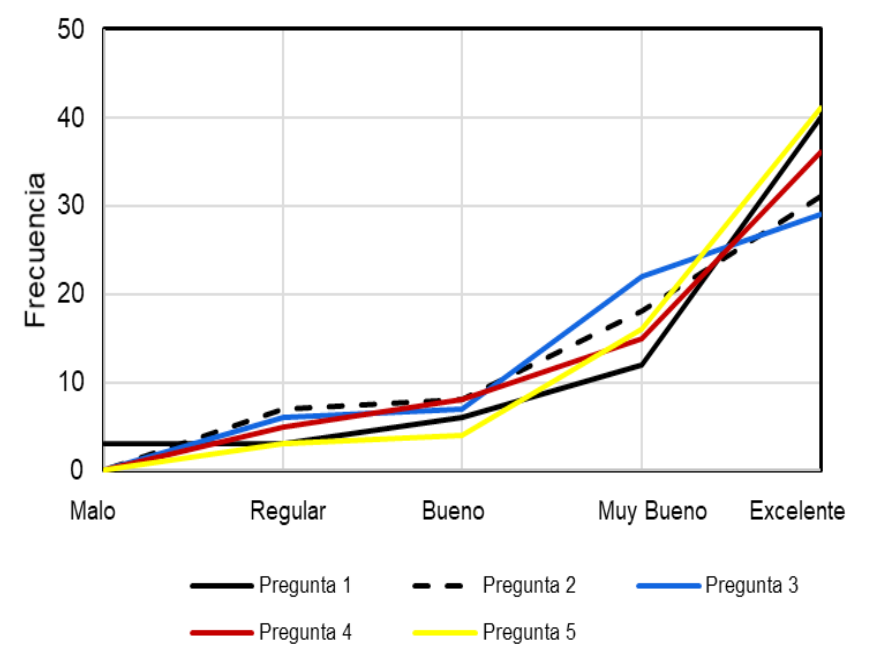

Fig. 6. Frecuencia de percepción de las cinco preguntas del cuestionario. Elaboración: Propia.

Por lo anterior, de aquí en adelante nos centramos en discutir las diferencias de las percepciones de las preguntas en las categorías muy bueno y excelente donde existe un rango más amplio de votaciones. A partir de estas diferencias podemos hacer algunas inferencias, lo que no pasa en las categorías de malo, regular y bueno. Las preguntas 2 (Califique la calidad de los simuladores con relación a la evaluación en línea.) y 3 (¿Cómo califica la oportunidad de mejorar su rendimiento académico mediante el EVA?) son las que obtuvieron menos votaciones en la categoría de excelente con $\mu=30$ estudiantes; una diferencia notoria con respecto a la $\mu=$ 39 estudiantes de las preguntas 1, 4 y 5 . Sin embargo, en la categoría de muy bueno las votaciones de las preguntas 2 y 3 obtienen los mayores puntajes, con $\mu=20$ estudiantes, la diferencia es menos marcada en las preguntas 1,4 y 5 con $\mu=16$ estudiantes. Finalmente, las preguntas 2 y 3 en las categorías muy bueno y excelente tienen una agrupación diferente a las otras preguntas, por lo tanto, estas dos preguntas tienen una alta relación entre sí, pero obtienen cuantificación diferente en los votos de percepción si comparamos con las otras preguntas.

\section{Conclusiones y perspectivas}

La percepción de los estudiantes sobre el uso de un EVA en su proceso de aprendizaje en educación secundaria fue que el uso es beneficioso y encuentran que las aplicaciones utilizadas durante el proceso son fáciles y funcionales, aunque existe un porcentaje reducido que percibe como malo este proceso. Como es de esperar, la mayoría de los estudiantes prefieren utilizar los recursos de las TICs (celulares, tablets); sin embargo, todavía hay una parte de la población estudiantil que, a pesar de la aparición de las tecnologías actuales, no está convencida de los beneficios de este tipo de entorno (estudiantes con percepción regular).

Concluimos que existe gran predisposición de estudiantes de educación secundaria para utilizar herramientas tecnológicas en EVA. En resumen, el EVA no es una panacea, pero puede ofrecer un ambiente de aprendizaje que puede complementar perfectamente el trabajo tradicional en el aula. En cuanto a las desventajas, se nuestra experiencia nos dice que la capacidad tecnológica de algunas instituciones educativas fiscales sigue siendo una amenaza para la aplicación de un EVA, y la distracción causada por las redes sociales y otros sitios de entretenimiento también puede constituir un impedimento para el proceso. Además, algunos profesores de nuestro medio siguen considerando que el uso de teléfonos móviles en clase es una amenaza, mientras que otros pueden utilizarlos como un recurso valioso para el proceso de aprendizaje de los estudiantes. Lamentablemente, no se pudo contar con una revisión a juicio de una persona con experiencia más extensa en el ámbito de la educación y recolección de datos. Esto, sin duda, hubiera reforzado la focalización y objetividad de las preguntas hacia los constructos de interés, sin embargo, las preguntas fueron cuidadosamente planificadas bajo el conocimiento y experiencia de los autores, aun así, se reconoce la falta de una revisión de expertos como un limitante a tener en cuenta en la realización de este estudio.

En conclusión, a pesar de las amenazas y limitaciones descritas anteriormente, puede afirmarse que la aplicación de un EVA en educación secundaria no sólo fue factible y funcional a lo largo de este estudio, sino que también puede ser implementada en otros procesos educativos. Los EVAs no pueden ser dependientes totalmente de TICs porque el riesgo de la pérdida de conexión a internet en ocasiones puede perjudicar el uso de un EVA, por lo tanto, es indispensable contar con medios alternos.

Las principales perspectivas de los EVAs son que los profesores están llamados a mantenerse al día con los 
avances en tecnología educativa, incluyendo EVAs y TICs para promover caminos de aprendizaje que se ajusten mejor a las necesidades e intereses de nuestros estudiantes de la era digital. Del mismo modo, se recomienda que los investigadores analicen cualitativa y cuantitativamente este campo con el fin de arrojar luz sobre el gran potencial para la fusión armoniosa y fructífera de la tecnología y el aprendizaje en la educación secundaria del Ecuador.

\section{Referencias}

Álvarez, F., Bartra, F., López, E., \& Sandoval, Y. (2015). Experiencias del Entorno Virtual de Aprendizaje en Ecuador. doi:10.13140/RG.2.1.2405.4804

Arroyo Vera, Z., Fernández Prieto, S., Barreto Zambrano, L., \& Paz Enrique, L. E. (2018). Entornos virtuales de aprendizaje en comunidades de práctica de docentes universitarios del Ecuador. Revista Ensayos Pedagógicos, 13(2), 185. doi:10.15359/rep.13-2.9

Barker, J., \& Peter, G. (2013). The Learning Impact of a Virtual Learning Environment: Students' views, 5(2).

de Lange, P., Suwardy, T., \& Mavondo, F. (2003). Integrating a virtual learning environment into an introductory accounting course: Determinants of student motivation. Accounting Education, 12(1), 1-14. doi:10.1080/0963928032000064567

Dyson, M., \& Barreto Campello, S. (2003). Evaluating Virtual Learning Environments: what are we measuring? Electronic Journal of E-Learning, 1(1), 1120.

Edel-Navarro, R. (2010). Entornos Virtuales de Aprendizaje. La contribución de "lo virtual" en la educación. Revista Mexicana de Investigación Educativa, 15(44), 7-15.

Hayes, B. E. (2009). Cómo medir la satisfacción del cliente: diseño de encuestas, uso y métodos de análisis estadístico. México, MX: Alfaomega.
Herrera Mosquera, L. (2017). Impact of implementing a virtual learning environment (VLE) in the EFL classroom. Ikala, 22(3), 479-498. doi:10.17533/udea. ikala.v22n03a07

Mueller, D., \& Strohmeier, S. (2011). Design characteristics of virtual learning environments: State of research. Computers and Education, 57(4), 2505-2516. doi:10.1016/j. compedu.2011.06.017

Rahmany, R., Sadeghi, B., \& Chegini, A. S. (2014). Normalization of CALL and TPACK: Discovering teachers' opportunities and challenges. Journal of Language Teaching and Research, 5(4), 891-900. doi:10.4304/jltr.5.4.891-900

Sneha, J. M., \& Nagaraja, G. S. (2014). Virtual Learning Environments-A Survey.

Taber, K. S. (2018). The Use of Cronbach's Alpha When Developing and Reporting Research Instruments in Science Education. Research in Science Education, 48(6), 1273-1296. doi:10.1007/s11165-016-9602-2

Vasquez, E., Nagendran, A., F. Welch, G., T. Marino, M., E. Hughes, D., Koch, A., \& Delisio, L. (2015). Virtual Learning Environments for Students with Disabilities: A Review and Analysis of the Empirical Literature and Two Case Studies. Rural Special Education Quarterly, 34(3), 26-32. doi:10.1177/875687051503400306

Virkus, S., Alemu, G. A., Demissie, T. A., Kokollari, B. J., Estrada, L. M. M., \& Yadav, D. (2009). Integration of digital libraries and virtual learning environments: A literature review. New Library World, 110(3-4), 136-150. doi:10.1108/03074800910941338

Walker, S. L., \& Fraser, B. J. (2005). Development and validation of an instrument for assessing distance education learning environments in higher education: The Distance Education Learning Environments Survey (DELES). Phenomenology and the Cognitive Sciences, 4(3), 289-308. doi:10.1007/s10984-005-1568-3

Copyright: (C) 2020 Urdiales-Flores, Armijos-Bacuilima \& Urdiales. Este es un artículo de acceso abierto distribuido bajo la licencia Creative Commons de Atribución No Comercial 4.0, que permite su uso sin restricciones, su distribución y reproducción por cualquier medio, siempre que no se haga con fines comerciales y el trabajo original sea fielmente citado. 